\title{
P02-5-14 Poster session
}

\section{The effects of histidine-rich glycoprotein on neutrophil-like differentiated cell lines}

\section{Yukinori Yoshii, Hidenori Wake, Kiyoshi Teshigawara, Keyue Liu, Dengli Wang, Yohei Takahashi, Masahiro Nishibori}

Pharmacology, Okayama University Graduate School of Medicine, Dentistry \& Pharmaceutical Sciences, Japan

Background: Excess immunothrombus formation induced by neutrophil adhesion on vascular wall, neutrophil extracellular traps (NETs) release and ROS production causes the multiple organ failure (MOF) in septic condition, leading to high mortality. Our previous study revealed that HRG treatment ameliorated the survival rate of septic mice due to suppressing the neutrophil adhesion, NETs release and immunothronbosis. These results suggested that HRG may be one of the most useful strategy for the treatment of septic patients, however, the effects of HRG on human purified neutrophil are not understood in detail. Human purified neutrophil can not survive for long time in vitro condition, and there are individual differences in the neutrophil response. Therefore, human neutrophils isolated from peripheral blood are not suitable for the long-term experiments. Thus, it's difficult to obtain the stable experiment system using neutrophils. All trans retinoic acid (ATRA) induces differentiation of the human myeloid leukemia cell lines HL-60 and NB-4. In the present study, we examined whether the differentiated myeloid cell line HL-60 and NB-4 showed the similar responses to human neutrophils purified from peripheral blood. Also, we investigate the effects of HRG on differentiated neutrophil-like cells in this study.

Method: HL-60 and NB-4 differentiated into a neutrophil lineage by ATRA. These cells were treated with Hank's Balanced Salt Solution (HBSS), human serum albumin (HSA), or HRG. The effects of HRG on these cells were evaluated on the basis of cell shape, extracellular ROS production, and microcapillary passage.

Result: HRG-treatoment kept the rounding shape of differentiated neutrophil-like cells and decreased the time to pass the cells through microcapillaries. These data indicated that HRG inhibits neutrophil adhesion on microcapillaries by maintaining the rounding cell shape. Moreover, this treatment suppressed the ROS production induced by TNF$\& \# 61537$; and LPS stimulations.

Conclusion: We showed that the ATRA-induced differentiated cell lines could be used as the alternative cells to investigate the effect of HRG on neutrophils. 